\title{
Clément Prévitali, 2014, Le sport à la campagne, Besançon, Presses Universitaires de Franche-Comté, 404 pages.
}

Sociologue, Clément Prévitali invite avec cet ouvrage issu de sa thèse de doctorat à une exploration originale de la fonction sociale et intégrative des associations sportives en milieu rural. En s'appuyant sur les apports d'une enquête au long cours dans la région Franche-Comté, laquelle combine usages des méthodes quantitatives - une enquête par questionnaires auprès d'un échantillon de 333 participants associatifs - et qualitatives - des entretiens avec des membres de vingt-et-une associations et de l'observation participante dans un club - l'auteur interroge ce qu'il voit, ce qu'il entend et ce qu'il connaît de ces instances. Pour ce faire, l'angle analytique des contacts sociaux et des liens sociaux qui s'opèrent, se construisent et se défont autour des associations est privilégié. Notamment inspiré, sur le plan conceptuel, des perspectives développées par Georges Simmel (1981) sur les influences et les déterminations que les individus exercent les uns sur les autres, l'auteur envisage ce type de dynamiques en empruntant, en parallèle, aux réflexions de Roger Brubaker (2001) sur l'identité. Cela est souligné au début du livre et à la suite de la préface écrite par Gilles Vieille Marchiset, la compréhension de cette activité sociale, de ses ressorts, de ses formes, de ses effets et de ses spécificités nécessite d'une part de replacer les associations sportives dans leur contexte géographique, spatial, démographique et social et, d'autre part, de caractériser les acteurs qui peuplent et animent les associations, à la fois sur le plan sportif - entraînement, compétition - et sur le plan extra-sportif - administratif, organisationnel, événementiel. Cette " plongée analytique " dans les associations sportives (p. 281) se structure en deux grandes parties. Intitulée «Les connectivités/dé-connectivités sportives au sein d'une configuration particulière », nourrie des apports de Norbert Elias (1991) sur l'interdépendance des individus, des groupes et des univers sociaux, la première partie consiste en une analyse des forces sociales et associatives ici en place. Une fois cet environnement reconstruit, l'auteur peut s'attacher, dans une deuxième partie titrée "Les connectivités sportives rurales. Un double processus d'intégration/exclusion ", d'une part à l'étude des modalités d'engagement au sein des associations sportives et, d'autre part, à l'examen du désengagement de ces instances. Cet aspect du retrait sera moins exploré que celui de l'investissement et de la présence dans les associations.

Dans une première partie, il s'agit donc d'exposer les caractéristiques des associations sportives et de leurs participants. Tandis que certaines comptent seulement une vingtaine d'adhérents, d'autres peuvent accueillir plusieurs centaines de personnes souvent résidentes de l'espace local ou installées dans les environs. À propos de ces «pratiquants ", « dirigeants » ou "spectateurs " (p. 63), au sein de clubs poursuivant des objectifs communs, à savoir la " pérennisation » (p. 87), l' « amélioration des infrastructures » et des "performances » (p. 89) et le maintien des effectifs bénévoles (p. 92), l'auteur souligne la prédominance de jeunes hommes mariés ou célibataires des milieux populaires non-agriculteurs (p. 60). Tandis que ces sportifs de sexe masculin 
réalisent des carrières longues dans cette pratique associative, "les sportives qui se lancent [...] le font plutôt sur le court terme » (p. 62). L'histoire de ces associations est le principe générateur et transformateur d'un « esprit club » (p. 97) caractérisé par des «marqueurs identitaires » (p. 100) et « un système de valeurs qui condense des manières de penser, d'agir, d'être » (p. 107). Ces éléments constituent l'association en une entité connue et reconnue, respectée et respectable. Ici, en général, au sein du club sportif, lequel fait souvent fonction de «deuxième famille » (p. 114), le loisir est préféré à la compétition (p. 116). Qui plus est, la «ruralité » (p. 120), c'est-à-dire, du point de vue de l'auteur, la convivialité et la solidarité caractéristiques du milieu rural et s'originant dans le passé agricole, est une valeur qui structure les relations sociales au sein des associations. Néanmoins, en quoi ces dynamiques sont-elles véritablement spécifiques à ce type d'environnement spatial et social ? Le texte n'apporte pas de réponse à cette question.

Une fois les conditions pratiques et symboliques de l'investissement associatif restituées, il s'agit, dans une deuxième partie, d'examiner les dynamiques d'intégration et d'exclusion liées aux « connectivités sportives rurales $»$ (p. 132). Tandis que les femmes paraissent essentiellement participer à des fins d' "épanouissement » et de «forme » physique, les hommes attendent surtout de cet engagement dont « l'objet sportif » fonde « la spécificité » (p. 145), " plaisir » et " convivialité » (p. 143). Les «modalités de participation » renforcent les « attaches relationnelles » (p. 172), favorisent la " groupalité ", le "sentiment d'appartenance à un groupe » (p. 173) et, par conséquent, l'« intégration »(p. 180). Irréductible à cette sociabilité, l'association existe aussi à travers ses relations avec les municipalités, lesquelles sont partie prenante, notamment dans le cadre des établissements publics de coopération intercommunale (EPCI), de " la construction d'un maillage social " à l'échelon communal (p. 187) décisif dans la recomposition de la société rurale. Les activités associatives internes et externes structurent le temps des participants, dont l'implication varie en fonction des évolutions de leurs vies "à côté " et des possibilités qu'ils ont de passer du temps et de dépenser de l'énergie dans les différentes dimensions de l'activité. Les plus investis sont les jeunes hommes célibataires et diplômés. Formant le «noyau dur » des participants à la "troisième mi-temps ", ils sont les "plus intégrés au groupe » (p. 207). Selon l'auteur, ce temps spécifique du soir « permet [...] de renforcer les attaches [....]. Il demeure également un indicateur important de la cohésion du groupe » (p. 223). L'association attire aussi ces participants vers l'organisation d'événements, une "implication qui n'est perçue à aucun moment comme une contrainte » (p. 230) et qui serait significative de "l'intériorisation de l'esprit club» (p. 238). Ici la solidarité, la camaraderie et l'amitié semblent régir les relations associatives, favoriser l'intégration des nouveaux venus et empêcher les conflits. Du moins ces derniers ne sont mentionnés ni par les enquêtés, ni par l'enquêteur, lequel avance, de notre point de vue peut-être trop rapidement, que les associations sportives urbaines sont l'envers de leurs homologues rurales sur le plan de la « convivialité ». 
Pour autant, il n'est pas question ici de dresser un tableau totalement idyllique de ces associations et, partant, de considérer qu'elles constituent nécessairement une zone confortable d'inscription sociale. En effet, tout en mettant l'accent sur leurs effets intégrateurs, l'auteur pointe aussi la force d'exclusion et de maintien à distance que les associations exercent parfois. Les participants ne sont pas nécessairement engagés à vie dans l'association qu'ils privilégient pendant une séquence donnée de leur trajectoire. Les problèmes de santé, les mutations professionnelles mais aussi les modifications de situation matrimoniale et les ruptures conjugales favorisent le désengagement temporaire ou définitif. Par ailleurs, « la profession peut représenter un frein à l'intégration des individus dans les clubs » (p. 288). Clément Prévitali prend l'exemple d'un gendarme pour lequel cette identité professionnelle pose problème à ses condisciples associatifs. Un " déficit d'intériorisation de l'esprit club " (p. 294), un comportement désajusté "à celui préconisé " (p. 295), notamment en termes d'implication dans le domaine extra-sportif, peuvent conduire jusqu'à des départs décidés par les sportifs déçus ou alors forcés par la direction de l'association. L'auteur met ainsi au jour les limites de ces instances dans leur pouvoir intégrateur : " des membres déjà en place [...] ne souhaitent pas spécialement s'ouvrir aux individus extérieurs» (p. 301). Ces ratés d'intégration, ces incompatibilités, ne sont pas éclairés par les caractéristiques sociales et les couleurs de peau des différentes parties. Les rejets et les décalages identifiés ont-ils à voir avec des rapports de force entre catégories et groupes sociaux ? Les " discussions tendues en $3^{\mathrm{e}}$ mi-temps $»(\mathrm{p}$. 318) sont-elles liées à des processus de cet ordre?

Fondé sur les apports d'une enquête au sein de cinq communes de moins de 2000 habitants qualifiables, compte tenu de leur localisation, de « village isolé profond » (p. 44), de «village isolé relais » (p. 45), de « village périurbain profond " (p. 46), de «village isolé proche » (p. 47) et, enfin, de «village périurbain proche » (p. 48), le livre se présente comme étant très attentif à l'hétérogénéité des mondes ruraux. Au final, les mises en relation entre pratiques et représentations du sport, d'un côté, et contexte rural spécifique, de l'autre, sont peu présentes dans l'analyse. Par ailleurs, l'ouvrage annonce une préoccupation pour la diversité des associations rurales sportives sur le plan des disciplines offertes à la pratique, une diversité à prendre au sérieux pour saisir les disparités dans le rapport construit à cet espace associatif. Or, qu'il s'agisse d'évoquer les modes d'engagement dans les compétitions, les entraînements et les dimensions administrative et festive au sein des associations, Clément Prévitali relie assez peu ce qu'il entend au domaine sportif dont il est question et, partant, aux éléments qui structurent chaque type d'activité d'un point de vue sportif, compétitif, administratif, social et culturel. Alors les spécificités liées à l'inscription dans un sport dit « individuel » ou « collectif », « cher » ou " accessible ", dit " populaire » ou " bourgeois ", ne sont la plupart du temps considérées que de façon allusive. Très attentive aux situations matrimoniales, également soucieuse de reconstruire les parcours géographiques et associatifs 
des enquêtés, l'analyse aurait pu, de manière à éclairer encore davantage les discours, développer une préoccupation identique pour les origines et les appartenances sociales. Cette question de l'objectivation des choses entendues par la caractérisation des acteurs amène à une autre remarque, cette fois-ci d'ordre méthodologique. Si les apports quantitatifs issus d'une enquête par questionnaires et les apports qualitatifs offerts par les entretiens réalisés par l'auteur sont largement mobilisés, en revanche le rendu de l'observation participante au sein d'un club de football n'apparaît pas explicitement dans le texte. Il s'agit moins de mettre en doute l'engagement ethnographique de l'auteur que de regretter l'absence de restitution de scènes saisies in situ au moins aussi instructives que les entretiens pour apprendre ce qui se joue sur les terrains de jeux, dans les vestiaires et dans les clubs-house. «À travers sa socialisation, puis par sa trajectoire et son expérience au sein du club, tout adhérent acquiert des dispositions, des schémas d'action ou de perception lui donnant l'occasion d'incorporer un ensemble de manières de penser, de sentir et d'agir [...] qui se révèlent durables » écrit l'auteur (p. 352). Parce qu'il prend le parti de mobiliser davantage les questionnaires et les entretiens que les observations, l'auteur fonde essentiellement cette analyse de processus socialisateurs sur les discours des enquêtés. Compte tenu de la difficulté à identifier les effets de cette participation sur cette seule base empirique, ces propositions eurent probablement gagné en force si elles avaient été étayées, par ailleurs, à la fois par des observations rendant compte des pratiques qui favorisent cette incorporation et par des analyses biographiques détaillées restituant les étapes de ces acquisitions de dispositions. L'analyse de cette «société flottante » (p. 354) des associations sportives gagnerait également, dans une étape ultérieure de cette réflexion d'envergure sur la vie rurale, à une prise de distance plus importante à l'égard des discours, discours auquel l'auteur conferre parfois le statut d'analyses. Cette distanciation procéderait par une restitution aux lecteurs des ressorts biographiques, académiques, financiers et géographiques de la recherche.

Au total, ces observations sur l'exploitation et l'exposition des matériaux empiriques n'enlèvent rien à l'intérêt de l'étude menée par Clément Prévitali, lequel explore des univers sociaux dont peu de sociologues ont considéré jusqu'à présent qu'ils valaient autant d'investissement scientifique. Cette approche "configurationnelle " de la vie des associations sportives rurales est ambitieuse dans le sens où elle complexifie à l'extrême le traitement sociologique du sport associatif rural. Comme il est peu aisé d'embrasser dans un même mouvement les dynamiques territoriales, culturelles, sociales, genrées, politiques et administratives qui pèsent sur ce domaine, cette complexification impose parfois une certaine superficialité analytique. Force est de constater, néanmoins, que ce livre élargit considérablement le champ des possibles empiriques et théoriques d'une sociologie rurale du sport pleinement connectée d'une part avec l'analyse des politiques publiques 
territoriales et, d'autre part, avec le savoir sur l'hétérogénéité des conditions rurales.

Sylvain BORDIEC

LACES, Université de Bordeaux

sylvain.bordiec@u-bordeaux.fr

\section{Bibliographie}

Brubaker R. (2001) Au-delà de l'identité, Actes de la recherche en sciences sociales, 139, 66-85.

Elias N. (1991) Qu'est-ce que la sociologie?, L'Aube, La Tour d'Aigues, France, $222 \mathrm{p}$.

Simmel G. (1981) Sociologie et Épistémologie, PUF, Paris, France, 238 p. 
